\title{
Revista Colombiana de

\section{Indicadores de calidad en la atención de pacientes con infarto agudo de miocardio}

\author{
Mariana Soto $^{\mathrm{a}}$, Andrés F. Buitrago ${ }^{\mathrm{a}, \mathrm{b}, *}$, Mabel Gómez ${ }^{\mathrm{a}, \mathrm{b}}$ y Édgar Celis ${ }^{\mathrm{a}, \mathrm{c}}$ \\ a Departamento de Medicina Crítica y Cuidado Intensivo, Hospital Universitario Fundación Santa Fe de Bogotá, Bogotá, Colombia \\ b Departamento de Medicina Interna, Sección de Cardiología, Hospital Universitario Fundación Santa Fe de Bogotá, Bogotá, \\ Colombia \\ c Departamento de Anestesia, Hospital Universitario Fundación Santa Fe de Bogotá, Bogotá, Colombia
}

Recibido el 20 de febrero de 2014; aceptado el 3 de julio de 2014

Disponible en Internet el 26 de noviembre de 2014

\section{PALABRAS CLAVE Infarto agudo de miocardio; Servicio de cardiología; Registro}

\begin{abstract}
Resumen
Objetivo: Describir el cumplimiento de los indicadores de calidad en la atención del infarto agudo de miocardio en un hospital de cuarto nivel en Bogotá, Colombia.

Métodos: Estudio observacional, retrospectivo, descriptivo, en el que se incluyeron todos los pacientes con diagnóstico de infarto agudo de miocardio, de acuerdo con la Tercera Definición Universal del Infarto, que ingresaron a la Fundación Santa Fe de Bogotá desde enero de 2011 hasta abril de 2013.

Resultados: El 99\% de los pacientes recibió aspirina al ingreso y al 98\% se le formuló durante el egreso. El $97 \%$ de los pacientes tuvo valoración intrahospitalaria de la fracción de eyección. El 93\% fue dado de alta con betabloqueador y el $88 \%$ con inhibidores de la enzima convertidora de angiotensina o antagonistas del receptor de angiotensina ॥. El 98\% recibió orden de rehabilitación cardiaca. La mortalidad por cualquier causa fue del $6 \%$.

Conclusiones: Existe adherencia adecuada a los indicadores de calidad en la atención del infarto agudo de miocardio, comparable con estándares internacionales, hecho del que hasta el momento no se tiene documentación alguna en Colombia.

(c) 2014 Sociedad Colombiana de Cardiología y Cirugía Cardiovascular. Publicado por Elsevier España, S.L.U. Todos los derechos reservados.
\end{abstract}

\footnotetext{
* Autor para correspondencia.

Correo electrónico: abuitrag@uniandes.edu.co (A.F. Buitrago).
} 


\section{KEYWORDS}

Myocardial acute

infarction;

Cardiology

department;

Registries

\section{Quality indicators in the care of patients with acute myocardial infarction}

\begin{abstract}
Objective: Describe the quality of care in patients presenting with acute myocardial infarction in a fourth level hospital in Bogotá, Colombia.

Methods: Observational, retrospective, descriptive study. From January 2011 to April 2013, all patients arriving to Hospital Fundación Santa Fe de Bogotá with acute myocardial infarction according to the Third Universal Definition of Myocardial Infarction were included.

Results: Aspirin at arrival was given to $99 \%$ of patients. Aspirin at discharge was given to $98 \%$. Evaluation of left ventricular ejection fraction was performed in $97 \%$ of patients. Ninety three percent received beta-blocker at discharge, $88 \%$ received angiotensin-converting enzyme inhibitors or angiotensin receptor blockers. Ninety eight percent were enrolled in a cardiac rehabilitation program. All-cause mortality was $6 \%$.

Conclusions: Quality performance indicators are fulfilled and our results are comparable to those of international standards. Actually there is no record of this information in Colombia. (C) 2014 Sociedad Colombiana de Cardiología y Cirugía Cardiovascular. Published by Elsevier España, S.L.U. All rights reserved.
\end{abstract}

\section{Introducción}

En la actualidad, el campo de la medicina experimenta cada vez más un mayor interés por cuantificar y mejorar la calidad en la atención de los pacientes ${ }^{1}$. Este interés probablemente sea el resultado de la transformación reciente pero dramática que han tenido los sistemas de salud, junto con cambios en la organización estructural y en las estrategias de reembolso que, en última instancia, pueden afectar la calidad de la atención ${ }^{2}$. Si bien la calidad de la atención es un tema que debe abordarse desde una perspectiva multidisciplinaria en cuanto a términos de seguridad, equidad y eficiencia, entre otros, tener medidas que permitan cuantificarla es un aspecto clave para identificar las prácticas clínicas que llevan a mejorarla.

Las guías de práctica clínica se construyen a partir de recomendaciones con diferentes grados de evidencia; no obstante, hay ocasiones en las que la evidencia que respalda un proceso es tan fuerte que omitir esta medida puede perjudicar el desenlace de un paciente ${ }^{1}$. Cuantificar la adherencia a estos procesos actúa como medida directa de la calidad de la atención; además, los programas de mejoría de la calidad de la atención potencian la adherencia de los hospitales a las guías de práctica clínica y los desenlaces de los pacientes ${ }^{1,3}$. Infortunadamente, en la mayoría de países no se cuenta con un sistema nacional que pueda documentar la calidad de la atención que se brinda a los ciudadanos. Los datos reportados en la literatura indican que no existe documentación apropiada en los sistemas de salud del tratamiento de las principales enfermedades, falta evaluación sistemática de los desenlaces, existen variaciones en el manejo de pacientes con patologías similares por parte de los prestadores de salud y hay ausencia de sistemas de monitoreo ${ }^{2}$.

No obstante, prevalecen algunos indicadores de calidad creados por otros grupos. En la medida en que se utilicen indicadores similares puede crearse sinergia con estas medidas ya existentes con el fin de minimizar la variabilidad de los datos al momento de reportar estos indicadores. Los centros de los servicios de Medicare y Medicaid en Estados Unidos, la Joint Commission on Accreditation of Healthcare Organizations, el American College of Cardiology y la American Heart Association, entre otros, son grupos que han definido y revisado indicadores en la atención del infarto agudo de miocardio ${ }^{1}$.

En Colombia la enfermedad cardiovascular es la causa principal de mortalidad en la población general. La Organización Panamericana de la Salud reporta que en este país las enfermedades isquémicas del corazón son responsables del $18 \%$ de las muertes en personas de 35 a 95 años, o más, con una tasa de mortalidad de tendencia ascendente ${ }^{4,5}$. Las guías de práctica clínica se desarrollaron con el fin de guiar a los proveedores de salud en el cuidado del paciente con base en la evidencia. Hasta el momento en Colombia no se han desarrollado indicadores que permitan evaluar la adherencia de los proveedores de salud a las guías de manejo de pacientes con infarto agudo de miocardio y, por tanto, no se tiene información sobre el porcentaje del cumplimiento de las recomendaciones basadas en la evidencia en lo que respecta a la atención de estos pacientes. El objetivo de este artículo es describir el cumplimiento de los indicadores de calidad en la atención del infarto agudo de miocardio en un hospital de cuarto nivel en Bogotá, Colombia.

\section{Metodología}

Se llevó a cabo un estudio observacional, retrospectivo, descriptivo, en el que se incluyeron todos los pacientes con diagnóstico de infarto agudo de miocardio de acuerdo con la Tercera Definición Universal del Infarto (tabla 1) que ingresaron a la Fundación Santa Fe de Bogotá desde enero de 2011 hasta abril de $2013^{6}$. El protocolo fue revisado y aprobado por el comité institucional de ética médica. La información de cada paciente se recolectó a partir de datos de la historia clínica. 
Tabla 1 Tercera Definición Universal de Infarto

\begin{tabular}{|c|c|}
\hline Tipo & Definición \\
\hline 1 & $\begin{array}{l}\text { Infarto de miocardio espontáneo relacionado } \\
\text { con rotura, ulceración o erosión de una placa } \\
\text { aterosclerótica, con la formación subsecuente } \\
\text { de trombo intramural en una o más arterias } \\
\text { coronarias llevando a disminución del flujo } \\
\text { sanguíneo o a formación de émbolo } \\
\text { plaquetario distal con necrosis miocárdica }\end{array}$ \\
\hline 2 & $\begin{array}{l}\text { Infarto de miocardio por disbalance entre el } \\
\text { aporte y/o demanda de oxígeno al miocardio } \\
\text { (ejemplo: disfunción endotelial coronaria, } \\
\text { espasmo coronario, arritmias, anemia, falla } \\
\text { respiratoria, hipotensión, hipertensión) }\end{array}$ \\
\hline 3 & $\begin{array}{l}\text { Muerte cardiaca con síntomas sugestivos de } \\
\text { isquemia miocárdica y presuntos cambios } \\
\text { electrocardiográficos o nuevo bloqueo de rama } \\
\text { izquierda, pero la muerte ocurre antes de que } \\
\text { se puedan tomar muestras sanguíneas, antes } \\
\text { de que se eleven las enzimas cardiacas o } \\
\text { cuando no se tomaron muestras sanguíneas } \\
\text { (casos raros) }\end{array}$ \\
\hline $4 a$ & $\begin{array}{l}\text { Infarto de miocardio asociado con intervención } \\
\text { cardiaca percutánea }\end{array}$ \\
\hline $4 b$ & $\begin{array}{l}\text { Infarto de miocardio asociado con trombosis } \\
\text { del stent }\end{array}$ \\
\hline 5 & $\begin{array}{l}\text { Infarto de miocardio asociado con cirugía } \\
\text { de revascularización miocárdica }\end{array}$ \\
\hline
\end{tabular}

Fuente: Thygesen et al. ${ }^{6}$.

\section{Definición de indicadores}

La calidad de atención en salud se define como el grado en el que los sistemas de salud aumentan la probabilidad de obtener los resultados deseados para los pacientes y poblaciones, y son coherentes con el conocimiento actual de los profesionales de la salud. Los indicadores son una de las formas actuales con las que se cuantifica la calidad de atención en salud. Son medidas cuantitativas que evalúan un proceso o desenlace específico en salud y que se utilizan como método de monitorización y evaluación de funciones que afectan los desenlaces de los pacientes. Su utilidad no radica únicamente en la evaluación de la calidad de la atención en salud, ya que también son útiles para realizar comparaciones entre diferentes hospitales, establecer prioridades, apoyar los procesos de regulación y acreditación y respaldar las elecciones de los pacientes en cuanto a proveedores de salud ${ }^{2}$.

Los indicadores de calidad pueden dividirse en indicadores de estructura, proceso o resultado. En este artículo se tendrán en cuenta los indicadores de proceso, que miden lo que se hace o se deja de hacer en el ámbito clínico, con énfasis en que la adherencia a la evidencia científica debe guiar las decisiones que se toman respecto a los pacientes. Este tipo de indicadores son los más usados por organizaciones como la Joint Commission on Accreditation of Healthcare Organizations para los procesos de acreditación de las instituciones. Finalmente, los indicadores de resultado analizan la calidad de los resultados, que están influenciados por muchos factores que no siempre son modificables por parte del equipo de salud (sexo, edad, severidad de la enfermedad, comorbilidades, riesgo previo entre otros) y por ende tienen limitaciones en su medición y no siempre se puede garantizar que su resultado esté libre de azar o confusiones ${ }^{2}$.

La selección de los indicadores de calidad se basó en publicaciones previas realizadas por expertos en el área ${ }^{1,7}$. Se definieron el total de pacientes elegibles para cada indicador (denominador) y el total de pacientes que cumplieron con el mismo (numerador). Los indicadores y criterios de elegibilidad se explican en la tabla 2.

\section{Análisis estadístico}

Las variables continuas se describieron por medio de estadísticos de tendencia central y de dispersión, utilizando media y desviación estándar. Las variables discretas se describieron a través de frecuencias y porcentajes. Los datos se analizaron mediante el paquete estadístico STATA 12 (Data analysis and Statistical Software).

\section{Resultados}

Desde enero de 2011 hasta abril de 2013 se incluyeron 324 pacientes, de los cuales un 64,20\% fueron hombres con edad media de 65,8 años. En la tabla 3 se describen las características de la población.

La mayoría presentó antecedente de infarto agudo de miocardio. Adicionalmente, los antecedentes más frecuentes fueron hipertensión arterial, en un 56\%, y dislipidemia, en un $44 \%$.

La presentación clínica del infarto fue principalmente sin elevación del segmento ST, en el 62\%. El 81\% tuvo una presentación clínica de infarto tipo 1. La mayoría acudió con clase Killip-Kimball I (85\%), y el 4\% en shock cardiogénico.

Casi todos recibieron aspirina en las primeras $24 \mathrm{~h}$ de hospitalización (99\%); así mismo, la mayoría fueron dados de alta con indicación de uso crónico de la misma (98\%). Durante la hospitalización, el $97 \%$ tuvo una valoración de la fracción de eyección del ventrículo izquierdo por medio de ecocardiograma. El 93\% fue dado de alta con betabloqueador. De aquellos con fracción de eyección menor o igual al $40 \%$, el $88 \%$ fueron dados de alta con inhibidores de la enzima convertidora de angiotensina o antagonistas del receptor de angiotensina ॥. Al 98\% se les dio orden ambulatoria de rehabilitación cardiaca. Finalmente, la mortalidad por cualquier causa fue del $6 \%$. Todos los pacientes incluidos en el numerador y denominador se exponen en la tabla 4 . El porcentaje de cumplimiento se ve reflejado en la figura 1.

\section{Discusión}

La importancia de evaluar la calidad en la atención de la salud no se discute. Para esto se han desarrollado indicadores que permiten medir la estructura, el proceso y el resultado de la atención de los pacientes. Ya existen indicadores que se usan en todo el mundo para este efecto y que permiten la comparación de los centros de atención entre ellos mismos.

En este estudio se midieron principalmente indicadores de proceso y se tuvieron los datos del registro ACTION del 
Tabla 2 Definición de indicadores de calidad de infarto agudo de miocardio

\begin{tabular}{ll}
\hline Indicador & Numerador \\
\hline Aspirina al ingreso & Pacientes con infarto agudo de \\
& miocardio que reciben aspirina \\
& en las primeras $24 \mathrm{~h}$ de la \\
& llegada al hospital
\end{tabular}

Aspirina al egreso

Betabloqueadores al egreso

\section{IECA/ARA II para disfunción ventricular izquierda}

Evaluación de la función sistólica del ventrículo izquierdo durante la hospitalización

Denominador

Pacientes $\geq 18$ años que ingresaron a la unidad de cuidados intensivos con diagnóstico de infarto agudo de miocardio según la Tercera Definición Universal de Infarto Población excluida:

-Pacientes < 18 años

-Pacientes remitidos de otra institución

-Pacientes con limitación del esfuerzo terapéutico

-Pacientes que rechazaron el tratamiento médico

-Pacientes que fallecieron en las primeras $24 \mathrm{~h}$

- Pacientes que fueron dados de alta en las primeras $24 \mathrm{~h}$

-Pacientes con contraindicación documentada en la historia clínica

Pacientes con infarto agudo de miocardio a quienes se les formula aspirina al alta para manejo ambulatorio

Pacientes con infarto agudo de miocardio a quienes se les formula betabloqueador al alta para manejo ambulatorio

Pacientes con infarto agudo de miocardio y disfunción ventricular izquierda ( $\mathrm{FEVI} \leq 40 \%)$ a quienes se les formula IECA/ARA II al alta para manejo ambulatorio

Pacientes con infarto agudo de miocardio a quienes se les evalúa la función ventricular sistólica del ventrículo izquierdo durante la hospitalización
Pacientes $\geq 18$ años que egresaron del hospital con diagnóstico de infarto agudo de miocardio según la Tercera Definición Universal de Infarto

Población excluida:

-Pacientes < 18 años

- Pacientes remitidos a otra institución

-Pacientes con limitación del esfuerzo terapéutico

-Pacientes que rechazaron el tratamiento médico

-Pacientes que fallecieron

-Pacientes con contraindicación documentada en la historia clínica

Pacientes $\geq 18$ años que egresaron del hospital con diagnóstico de infarto agudo de miocardio según la Tercera Definición Universal de Infarto

Población excluida:

-Pacientes < 18 años

-Pacientes remitidos a otra institución

-Pacientes con limitación del esfuerzo terapéutico

-Pacientes que rechazaron el tratamiento médico

-Pacientes que fallecieron

-Pacientes con contraindicación documentada en la historia clínica

Pacientes $\geq 18$ años que egresaron del hospital con diagnóstico de infarto agudo de miocardio según la Tercera Definición Universal de Infarto y disfunción ventricular izquierda determinada por $\mathrm{FEVI} \leq 40 \%$

Población excluida:

-Pacientes < 18 años

-Pacientes remitidos a otra institución

-Pacientes con limitación del esfuerzo terapéutico

-Pacientes que rechazaron el tratamiento médico

-Pacientes que fallecieron

-Pacientes con contraindicación documentada en la historia clínica para IECA y ARA II

Pacientes $\geq 18$ años que ingresaron a la unidad de cuidados intensivos con diagnóstico de infarto agudo de miocardio según la Tercera Definición Universal de Infarto Población excluida:

-Pacientes < 18 años

-Pacientes remitidos a otra institución

-Pacientes con limitación del esfuerzo terapéutico

-Pacientes que rechazaron el tratamiento médico

-Pacientes que fallecieron

- Pacientes con razones médicas documentadas en la historia clínica para no realizar evaluación de la función sistólica del ventrículo izquierdo 
Tabla 2 (continuación)

\begin{tabular}{|c|c|c|}
\hline Indicador & Numerador & Denominador \\
\hline $\begin{array}{l}\text { Rehabilitación } \\
\text { cardiaca }\end{array}$ & $\begin{array}{l}\text { Pacientes con infarto agudo de } \\
\text { miocardio a quienes se les da } \\
\text { indicación de asistir a un } \\
\text { programa de rehabilitación } \\
\text { cardiaca ambulatorio }\end{array}$ & $\begin{array}{l}\text { Pacientes } \geq 18 \text { años que ingresaron a la unidad de cuidados } \\
\text { intensivos con diagnóstico de infarto agudo de miocardio } \\
\text { según la Tercera Definición Universal de Infarto } \\
\text { Población excluida: } \\
\text {-Pacientes < } 18 \text { años } \\
\text {-Pacientes remitidos a otra institución } \\
\text {-Pacientes con limitación del esfuerzo terapéutico } \\
\text {-Pacientes que rechazaron el tratamiento médico } \\
\text {-Pacientes que fallecieron } \\
\text {-Pacientes con razones médicas documentadas en la historia } \\
\text { clínica para no realizar rehabilitación cardiaca ambulatoria }\end{array}$ \\
\hline $\begin{array}{l}\text { Mortalidad } \\
\quad \text { intrahospitalaria }\end{array}$ & $\begin{array}{l}\text { Pacientes que fallecen durante } \\
\text { la hospitalización con } \\
\text { diagnóstico de infarto agudo } \\
\text { de miocardio }\end{array}$ & $\begin{array}{l}\text { Pacientes } \geq 18 \text { años que ingresaron al hospital con diagnóstico } \\
\text { de infarto agudo de miocardio según la Tercera Definición } \\
\text { Universal de Infarto } \\
\text { Población excluida: } \\
\text {-Pacientes < } 18 \text { años } \\
\text {-Pacientes remitidos a otra institución }\end{array}$ \\
\hline
\end{tabular}

ARA II: antagonista del receptor de angiotensina॥; FEVI: fracción de eyección del ventrículo izquierdo; IECA: inhibidor de la enzima convertidora de angiotensina.

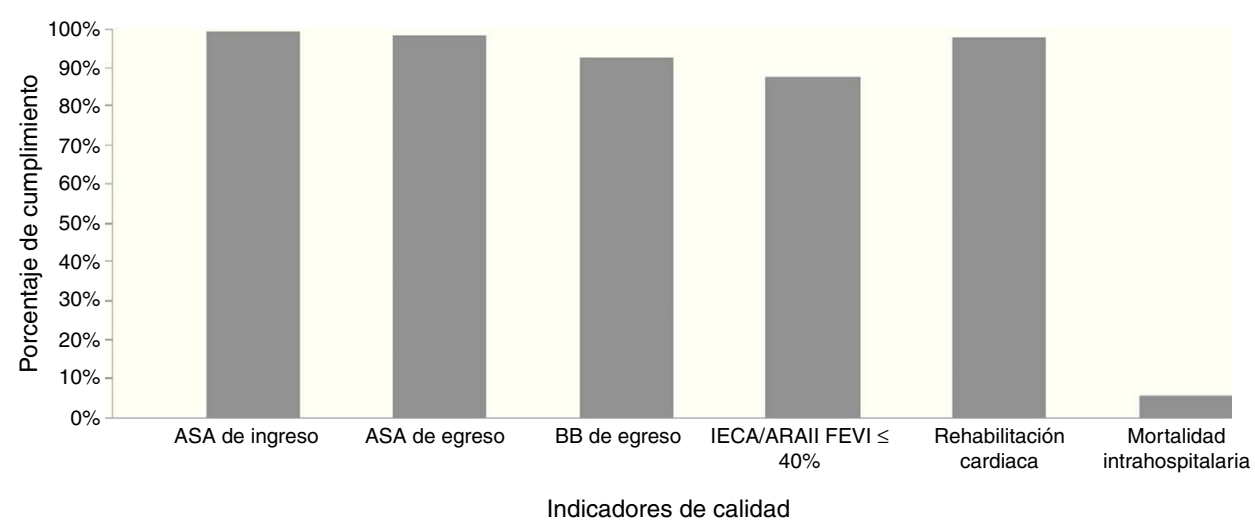

Figura 1 Porcentaje de cumplimiento de indicadores de calidad de infarto agudo de miocardio

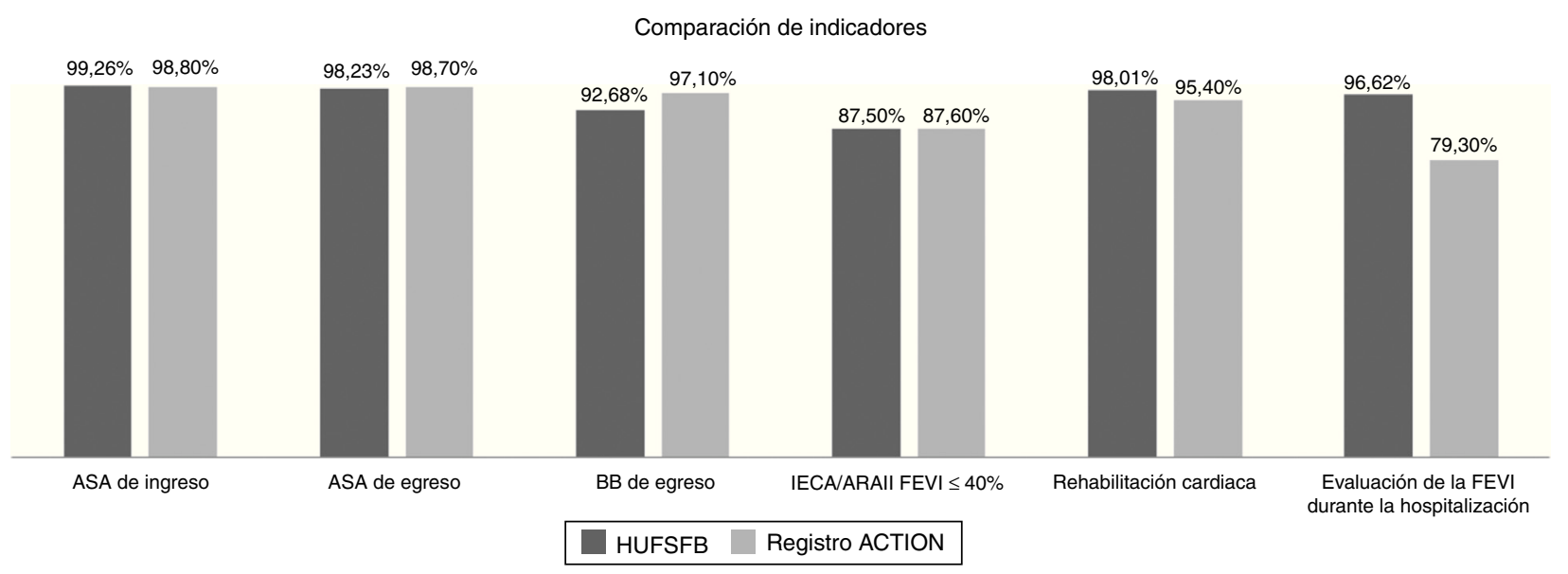

Figura 2 Comparación con indicadores internacionales. 
Tabla 3 Características de la población

\begin{tabular}{|c|c|c|}
\hline Variables & n (324) & $\%$ \\
\hline \multicolumn{3}{|l|}{ Características demográficas } \\
\hline Género masculino & 208 & 64,20 \\
\hline Género femenino & 116 & 35,80 \\
\hline Edad $\pm D E$, años & $65,8 \pm 13,74$ & \\
\hline \multicolumn{3}{|l|}{ Antecedentes } \\
\hline Hipertensión & 181 & 55,86 \\
\hline Diabetes & 66 & 20,37 \\
\hline Tabaquismo & 105 & 32,41 \\
\hline Dislipidemia & 142 & 43,83 \\
\hline Antecedente de IAM & 293 & 90,43 \\
\hline Antecedente de EAP & 5 & 1,54 \\
\hline Antecedente de ACV/AIT & 17 & 5,25 \\
\hline \multicolumn{3}{|l|}{ Presentación clínica } \\
\hline IAMCEST & 124 & 38,27 \\
\hline IAMSEST & 200 & 61,73 \\
\hline \multicolumn{3}{|l|}{ Tipo } \\
\hline 1 & 261 & 80,56 \\
\hline 2 & 53 & 16,36 \\
\hline 3 & 5 & 1,54 \\
\hline $4 a$ & 0 & 0,00 \\
\hline $4 b$ & 3 & 0,93 \\
\hline 5 & 2 & 0,62 \\
\hline \multicolumn{3}{|l|}{ Clase Killip-Kimball al ingreso } \\
\hline 1 & 274 & 84,57 \\
\hline$\|$ & 20 & 6,17 \\
\hline III & 17 & 5,25 \\
\hline IV & 13 & 4,01 \\
\hline
\end{tabular}

ACV: accidente cerebrovascular; AIT: accidente isquémico transitorio; EAP: enfermedad arterial periférica; IAM: infarto agudo de miocardio; IAMCEST: infarto agudo de miocardio con elevación del ST; IAMSEST: infarto agudo de miocardio sin elevación del ST.

National Cardiovascular Data Registry (NCDR) como punto de comparación con estándares internacionales (fig. 2).

En los pacientes con infarto agudo de miocardio, la administración de aspirina al ingreso tiene una evidencia $I B^{8}$ y se considera un indicador en la calidad de la atención. Se ha demostrado que el uso de aspirina al ingreso disminuye la mortalidad ${ }^{9}$. En este estudio se encontró que existe un adecuado cumplimiento de esta práctica clínica, con un porcentaje de cumplimiento similar al reportado en el registro ACTION. Así mismo, se ha comprobado que el uso de aspirina posterior al síndrome coronario agudo disminuye la recurrencia del infarto agudo de miocardio y la tasa de mortalidad, y las guías de práctica clínica recomiendan su uso con una evidencia $I A^{8,9}$. Se considera que la prescripción de aspirina al egreso es un indicador de calidad. En este estudio se encontró que existe un alto porcentaje de cumplimiento de este indicador, similar al del registro ACTION.

Por otra parte, el uso crónico de betabloqueadores ha demostrado una reducción del riesgo de isquemia recurrente y de la tasa de mortalidad en pacientes con antecedente de infarto agudo de miocardio ${ }^{9}$. En la actualidad tienen una recomendación basada en la evidencia $\mathrm{IlaB}^{8}$, y por esto se consideran como un indicador que mide la calidad de la atención. En este estudio el porcentaje de cumplimiento fue alto, e incluso supera el que se reporta en el registro ACTION. Sin embargo, a lo largo del estudio se pudo evidenciar que cuando en algunas ocasiones no se formula el betabloqueador, no existe una justificación explícita en la historia clínica, lo cual puede dar una falsa idea de un menor porcentaje de cumplimiento con este indicador. Una posible solución es capacitar a los médicos tratantes sobre la importancia del cumplimiento de los indicadores de calidad e invitarlos a dejar siempre documentada la razón por la cual no se formula un medicamento que es considerado como estándar de calidad.

La cuantificación de la fracción de eyección del ventrículo izquierdo posterior a un infarto agudo de miocardio es de suma importancia, ya que puede identificar la extensión del infarto y los candidatos a terapias específicas, además de predecir supervivencia en pacientes con disfunción ventricular ${ }^{9}$. Tiene una evidencia $\mathrm{IB}^{8}$. En este estudio se encontró un alto porcentaje de cumplimiento; no obstante, es un poco menor al reportado por el registro ACTION, lo que posiblemente se deba a que en este estudio solo se tuvieron en cuenta aquellos pacientes a quienes se les realizó una evaluación intrahospitalaria de la función ventricular, mientras que en el registro ACTION se admitieron no solo estos pacientes sino aquellos a quienes no se les realizó de manera intrahospitalaria pero se les dejó orden ambulatoria.

De otro lado, el uso de inhibidores de la enzima convertidora de angiotensina en pacientes con disfunción ventricular izquierda ha disminuido el riesgo de eventos vasculares y la mortalidad. Ante contraindicaciones o intolerancia se pueden utilizar los antagonistas del receptor de angiotensina $\|^{9}$. Su recomendación en las guías basadas en la evidencia es

Tabla 4 Indicadores de calidad

\begin{tabular}{llll}
\hline Indicador & Numerador & Denominador \\
\hline Aspirina al ingreso & 268 & 270 & 99 \\
Aspirina al egreso & 277 & 282 & 98 \\
Betabloqueadores al egreso & 266 & 287 & 93 \\
IECA/ARA II para disfunción ventricular izquierda & 21 & 24 & 88 \\
Evaluación de la FEVI durante la hospitalización & 314 & 325 & 97 \\
Rehabilitación cardiaca & 295 & 301 & 98 \\
Mortalidad intrahospitalaria & 18 & 322 & 6 \\
\hline
\end{tabular}

ARA II: antagonista del receptor de angiotensina; FEVI: fracción de eyección del ventrículo izquierdo; IECA: inhibidor de la enzima convertidora de angiotensina. 
IA y IB, respectivamente; se consideran como indicador de calidad $^{8}$. En este estudio se encontró que en general existe una adecuada prescripción de estos medicamentos al alta, con un porcentaje de cumplimiento cercano al del registro ACTION. Adicionalmente se observó que en ocasiones, cuando no se formulan estos medicamentos, no queda una justificación clara en la historia clínica, por lo cual se hace la misma propuesta que para la formulación de betabloqueadores. Es importante tener en cuenta que la cantidad de pacientes con disfunción ventricular izquierda en este estudio es pequeña; esto hace que por cada paciente que no recibe este medicamento en ausencia de contraindicación, el cambio que sufre el porcentaje de cumplimiento sea mayor.

Finalmente, parte del tratamiento integral de estos pacientes consiste en la asistencia a programas de rehabilitación cardiaca, que ha demostrado reducir la mortalidad por cualquier causa y el riesgo de reinfarto. Así mismo, ayudan a mejorar factores de riesgo para enfermedad cardiovascular, la capacidad de ejercicio y la calidad de vida de estos pacientes; adicionalmente, ayudan a asegurar el cumplimiento adecuado de las terapias basadas en la evidencia ${ }^{8}$. Recientemente se implementaron como un indicador de calidad con el fin de ayudar a los sistemas de salud a implementar pasos que optimicen la remisión de estos pacientes a programas de rehabilitación cardiaca. En este estudio se encontró que no solo existe un adecuado cumplimiento en cuanto a la formulación de la fase ॥ de la rehabilitación cardiaca, sino que además existe un alto porcentaje de cumplimiento de esta terapia durante la fase intrahospitalaria. No obstante, el porcentaje de cumplimiento puede mejorar respecto al del registro ACTION. Nuevamente esto puede estar relacionado con que en ocasiones no queda documentado en la historia clínica.

\section{Limitaciones del estudio}

En primer lugar, la información se obtuvo de historias clínicas en las que, en ocasiones, algunos datos no quedan registrados. Por otro lado, en el estudio se tuvieron en cuenta todos los tipos de infarto, no solo los relacionados con enfermedad y rotura de placas ateroscleróticas, o ambos. En la actualidad no existen guías para el manejo de pacientes con infarto no relacionado con enfermedad aterosclerótica, como podría ser el caso de los infartos tipo 2. No obstante, esto trató de controlarse por medio de una definición adecuada tanto del numerador como del denominador de cada uno de los indicadores de calidad.

\section{Conclusiones}

La tendencia actual en el área de la salud se está enfocando en crear medidas que permitan cuantificar la calidad de la atención en salud. Las guías de medicina basadas en la evidencia constituyen un elemento que acompaña al clínico en sus decisiones, mientras que los indicadores de calidad en la atención de salud se convierten en un aspecto que debe cumplirse. Estos indicadores no solo permiten tener medidas que cuantifican la calidad de la atención, sino la comparación en los ámbitos local, nacional e internacional. Su desarrollo, uso y cumplimiento deben ser una de las prioridades de los centros de atención de salud. En este estudio se adaptaron indicadores que se han usado previamente en el campo internacional y que, además, se utilizan para acreditación en excelencia. Los resultados obtenidos muestran una adecuada adherencia que es comparable con estándares internacionales y de la que, hasta el momento, no se tiene documentación alguna en Colombia. Este estudio no solo aporta resultados que no están disponibles en la actualidad, sino que además abre las puertas para trabajar en un área con potencial de mejora: la historia clínica.

«No es el más fuerte de las especies el que sobrevive, tampoco es el más inteligente el que sobrevive. Es aquel que es más adaptable al cambio.»

CHARLES DARWIN

\section{Conflicto de intereses}

Los autores manifiestan no tener conflictos de intereses.

\section{Bibliografía}

1. Spertus JA, Eagle KA, Krumholz HM, Mitchell KR, Normand SL. American College of Cardiology and American Heart Association Methodology for the selection and creation of performance measures for quantifying the quality of cardiovascular care. J Am Coll Cardiol. 2005; 45:1147-56.

2. Mainz J. Defining and classifying clinical indicators for quality improvement. Int J Qual Health Care. 2003;15:523-30.

3. Qian F, Ling FS, Deedwania P, Hernandez AF, Fonarow GC, Cannon $\mathrm{CP}$, et al. Care and outcomes of Asian-American acute myocardial infarction patients: Findings from the American Heart Association get with the guidelines - Coronary Artery Disease Program. Circ Cardiovasc Qual Outcomes. 2012;5:126-33.

4. Panamerican Health Organization [Internet]. Colombia. Causas principales de mortalidad en las Américas [consultado 15 May 2013]. Disponible en: http://ais.paho.org/phip/viz/mort causasprincipales_lt_oms.asp

5. Así Vamos en Salud [Internet]. Colombia. Gráfica - Tasa de Mortalidad por Enfermedades Isquémicas del Corazón - Georeferenciado [consultado 15 May 2013]. Disponible en: http://www. asivamosensalud.org/inidicadores/estado-de-salud/grafica.ver/ 21

6. Thygesen K, Alpert JS, Jaffe AS, Simoons ML, Chaitman BR, White HD. Third universal definition of myocardial infarction. Eur Heart J. 2012;33:2551-67.

7. The Joint Commission International [Internet]. United States of America. [c2011] International Hospital Inpatient Quality Measures [consultado 15 May 2013]. Disponible en: http://www. jointcommission.org/

8. The Task Force on the management of ST-segment elevation acute myocardial infarction of the European Society of Cardiology (ESC). Guidelines for the management of acute myocardial infarction in patients presenting with ST-segment elevation. Eur Heart J. 2012;33:2569-619.

9. Krumholz HM, Anderson JL, Bachelder BL, Fihn SD, Michael P, Masoudi FA, et al. ACC/AHA 2008 performance measures for adults with ST-elevation and nonST-elevation myocardial infarction: A report of the American College of Cardiology/American Heart Association Task Force on performance measures. Circulation. 2008;118:2596-648. 